\title{
Dipeptidyl peptidase-4 inhibitors as add-on therapy to insulin in patients with type 2 diabetes mellitus: a meta-analysis of randomized controlled trials
}

This article was published in the following Dove Press journal:

Diabetes, Metabolic Syndrome and Obesity: Targets and Therapy

Na Wang ${ }^{\prime}$
Tao Yang
Jie Li $^{2}$

Xianfeng Zhang ${ }^{2}$

'Department of Endocrinology, The Affiliated Hospital of Jining Medical University, Jining 272000, People's

Republic of China; ${ }^{2}$ The 4th Department of Psychiatry, Jining Psychiatric Hospital, Jining 272000, People's Republic of China
Purpose: Addition of the dipeptidyl peptidase-4 (DPP4) inhibitors to insulin in patients with type 2 diabetes mellitus (T2DM) may achieve better glycemic control. However, results of pilot randomized controlled trials (RCTs) are inconsistent. We aimed to perform a metaanalysis of RCTs to evaluate efficacy and safety of DPP4 inhibitors compared with placebo/ no treatment as add-on therapy to insulin in T2DM patients.

Materials and methods: Relevant studies were identified via a search of PubMed, Cochrane Library, and Embase databases. A fixed or random effect model was applied according to the heterogeneity.

Results: Overall, 22 RCTs with 6,957 T2DM patients were included. Addition of DPP4 inhibitors to insulin was associated with significantly reduced HbAlc as compared with controls (weighed mean difference [WMD]: $-0.54 \%, p<0.001$ ). The benefits of DPP4 inhibitors as add-on therapy on HbAlc were independent of study design, follow-up duration, categories of DPP4 inhibitors used, and using of fixed/adjustable insulin doses as indicated by predefined subgroup analyses. Moreover, addition of DPP4 inhibitors to insulin was associated with significantly reduced fasting blood glucose (WMD: $-0.47 \mathrm{mmol} / \mathrm{L}$, $p<0.001$ ), postprandial glucose at $2 \mathrm{hrs}$ (WMD: $-2.03 \mathrm{mmol} / \mathrm{L}, p<0.001$ ), and daily dose of insulin (WMD: $-2.73 \mathrm{U} / \mathrm{d}, p<0.001$ ), while body weight (WMD: $0.02 \mathrm{~g}, p=0.81$ ) or risk of symptomatic hypoglycemia (risk ratio: $0.92, p=0.37$ ) were not affected.

Conclusions: Addition of DPP4 inhibitors to insulin significantly improved the glycemic control in T2DM patients without further increasing the risk of weight gain and hypoglycemia. Keywords: dipeptidyl peptidase-4 inhibitors, insulin, add-on therapy, diabetes mellitus, meta-analysis

\section{Introduction}

The incidence of type 2 diabetes mellitus (T2DM) is increasing rapidly worldwide. ${ }^{1-3}$ Pathophysiologically, patients with T2DM are characterized by insulin resistance and lack of insulin secretion by the $\beta$ cells of the pancreatic islet. ${ }^{4,5}$ Although initial treatment with oral antidiabetic drugs (OADs) is effective for glycemic control in T2DM patients, with the deterioration of the $\beta$ cells of the pancreatic islet during the progression of the disease, exogenous insulin injection is eventually needed., ${ }^{6,7}$ Currently, multiple regimens of insulin injections are applied in clinical practice, such as basal insulin treatment, basal and premeal bolus insulin injections, and premix insulin treatments, and with which, satisfying glycemic control can be achieved in most T2DM patients. ${ }^{8-10}$ However, insulin treatment is associated with adverse events
Correspondence: $\mathrm{Na}$ Wang

Department of Endocrinology, The Affiliated Hospital of Jining Medical

University, No. 89 Guhuai Road, Jining

272000, People's Republic of China

Tel +8605372903399

Fax +8605372903399

Email wangna22838@I26.com 
including gain of body weight (BW) and hypoglycemia, which are related to the increase of the daily insulin dose. ${ }^{11,12}$ Therefore, exploring an optimized add-on therapy to insulin is of clinical significance for the improvement of glycemic control and reducing the risk of adverse events in T2DM patients with insulin injections.

Conventionally, many OADs exert their hypoglycemic efficacies via insulin-dependent mechanisms, such as stimulation of endogenous insulin secretion and improvement of peripheral insulin resistance. ${ }^{13-15}$ The dipeptidyl peptidase-4 (DPP4) inhibitors are a novel group of OADs which exert hypoglycemic efficacy via inhibiting the degradation of gastrointestinal incretins including glucagon-like peptide-1 (GLP-1) and glucose-dependent insulinotropic polypeptide (GIP) ${ }^{16,17}$ Increased GLP-1 and GIP resulted from DPP4 inhibiting, potentiate glucose-stimulated insulin secretion, which is complementary to the direct insulin injection in T2DM patients. ${ }^{18}$ Moreover, unlike GLP-1 receptor agonists, DPP4 inhibitors can be orally administered, do not reduce BW, and do not cause serious gastrointestinal adverse reactions. ${ }^{19,20}$ Therefore, theoretically, combination of DPP4 inhibitors and insulin treatment may exert beneficial effects in T2DM patients compared to intensive insulin therapy. In fact, some pilot randomized controlled trials (RCTs) have evaluated the efficacies of DPP4 inhibitors as add-on therapy to insulin in T2DM patients. ${ }^{21-42}$ However, the scales of these RCTs are generally small and the results of them are not always consistent. Although two previous metaanalyses were performed to evaluate the efficacy of the addition of DPP4 inhibitors to insulin therapy in T2DM patients, these two studies are with certain methodological flaws. ${ }^{43,44}$ One of them included RCTs with active OADs in the control arm, which makes it difficult to interpret the results. ${ }^{43}$ The other one only included RCTs with stable insulin regimens, and studies with insulin dose titration were excluded, leading to the missing of the important study data. ${ }^{44}$ In addition, some recently published studies were not included in the previous meta-analysis, and the influences of patient and study characteristics on the hypoglycemic efficacy of DPP4 inhibitors added-on to insulin have not been determined. Therefore, we aimed to perform an updated meta-analysis to evaluate the overall effects of DPP4 inhibitors as add-on therapy to insulin in T2DM patients. Furthermore, particular attention will be paid regarding the influences of study design, patient characteristics, and categories of DPP4 inhibitors on the glycemic control efficacy in T2DM patients receiving a combined therapy with DPP4 inhibitors and insulin.

\section{Methods}

This meta-analysis was designed and performed in accordance with the Preferred Reporting Items for Systematic Reviews and Meta-Analyses ${ }^{45}$ and the Cochrane Handbook guidelines. ${ }^{46}$

\section{Search strategy}

We searched the Medline (PubMed), Embase (Ovid), and CENTER (Cochrane Library) databases using the combination of the following terms: (1) "DPP4", "DPP-4", "dipeptidyl peptidase-4 inhibitors", "sitagliptin", “vildagliptin", "linagliptin”, “saxagliptin", “alogliptin”, "dutogliptin", “aemigliptin”, "anagliptin", "teneligliptin", "trelagliptin", or "omarigliptin"; (2) "insulin"; and (3) "random", "randomly", or "randomized". The date of the final database search was October 18,2018 . We limited the search to clinical studies in humans. The references of related original and review articles were manually searched for potential studies.

\section{Study selection}

Studies that met the following criteria were included: (1) full-length articles in English; (2) RCTs with a parallel design; (3) included patients with confirmed T2DM; (4) assigned patients to either an oral DPP4 inhibitor intervention group or a placebo or no treatment control group, combined with insulin therapy with or without background OADs; (5) with at least ten patients in each arm; (6) with treatment duration of at least eight weeks; and (7) data of at least one of the following outcomes could be extracted or estimated, including glycosylated hemoglobin (HbA1c), fasting blood glucose (FBG), postprandial glucose at $2 \mathrm{hrs}$ (PPG2h), daily insulin dose, changes of $\mathrm{BW}$, and the incidence of symptomatic hypoglycemia events. We applied no limitations to insulin regimens (basal only, basal and premeal bolus, or premix insulin) in this study. Review articles, preclinical studies, and studies comparing DPP4 inhibitors with other active OADs as add-on therapy to insulin were excluded.

\section{Data extraction and quality assessment}

Literature search, data extraction, and quality assessment were performed by two authors independently. Discrepancies were resolved by consensus with a third author. The Cochrane Risk of Bias Tool $^{46}$ was applied for study quality evaluation. 


\section{Statistical analysis}

The meta-analysis and statistical analysis were performed with RevMan (Version 5.1; Cochrane Collaboration, Oxford, UK) and Stata (version 12.0; Stata Corporation, College Station, TX) software. The primary outcome of this meta-analysis was the difference between changes of $\mathrm{HbAlc}$ from baseline in DPP4 inhibitors and controls. The secondary outcomes included changes of FBG, PPG2h, daily insulin dose, BW, and the incidence of hypoglycemia. The effect of a continuous variable was presented as a weighed mean difference (WMD) with the $95 \% \mathrm{CI}$, while for a categorized variable, a risk ratio (RR) with the 95\% CI was used. Heterogeneity was evaluated by Cochrane's $\mathrm{Q}$ test, ${ }^{46}$ and significant heterogeneity was considered if $p<0.10$. The $\mathrm{I}^{2}$ statistic, indicating the percentage of total variation across studies, was also calculated as a description of heterogeneity. ${ }^{47} \mathrm{~A}$ random effect model was used if significant heterogeneity was detected; otherwise, a fixed effect model was applied. For studies with the more than one interventional arm, multiple comparisons were considered and included in the meta-analysis separately. Subgroup analyses were performed to evaluate the potential influence of predefined study and patient characteristics on HbAlc, including study design, sample size, mean age of the patients, followup duration, whether titration of insulin dose was applied, and which DPP4 inhibitor was used. Publication bias was assessed by visual inspection of the symmetry of the funnel plot $^{48}$ and Egger's regression asymmetry test. ${ }^{46} P$-values were twotailed, and statistical significance was set at $P<0.05$.

\section{Results}

\section{Study selection}

The process of study selection is summarized in Figure 1. Briefly, 1,851 studies were obtained via initial database search, and 51 potentially relevant studies were retrieved after selection based on titles and abstracts. Subsequently, 22 studies $^{21-42}$ were included in the meta-analysis after further excluding of 29 studies based on the following reasons: six were not RCTs, eight were not with addition of DPP4 inhibitors to insulin as intervention, eight were with follow-up durations $<8$ weeks, one was with $<10$ patients in each study arm, two were with combination of active OADs (sulfonylureas or glinides) and insulin in controls, and the other four were repeated reports of the included RCTs.

\section{Study characteristics and quality evaluation}

Overall, 22 RCTs with 6,957 T2DM patients were included in the meta-analysis. $^{21-42}$ One study ${ }^{22}$ included two intervention arms with alogliptin $12.5 \mathrm{mg} / \mathrm{d}$ and $25 \mathrm{mg} / \mathrm{d}$, respectively, and

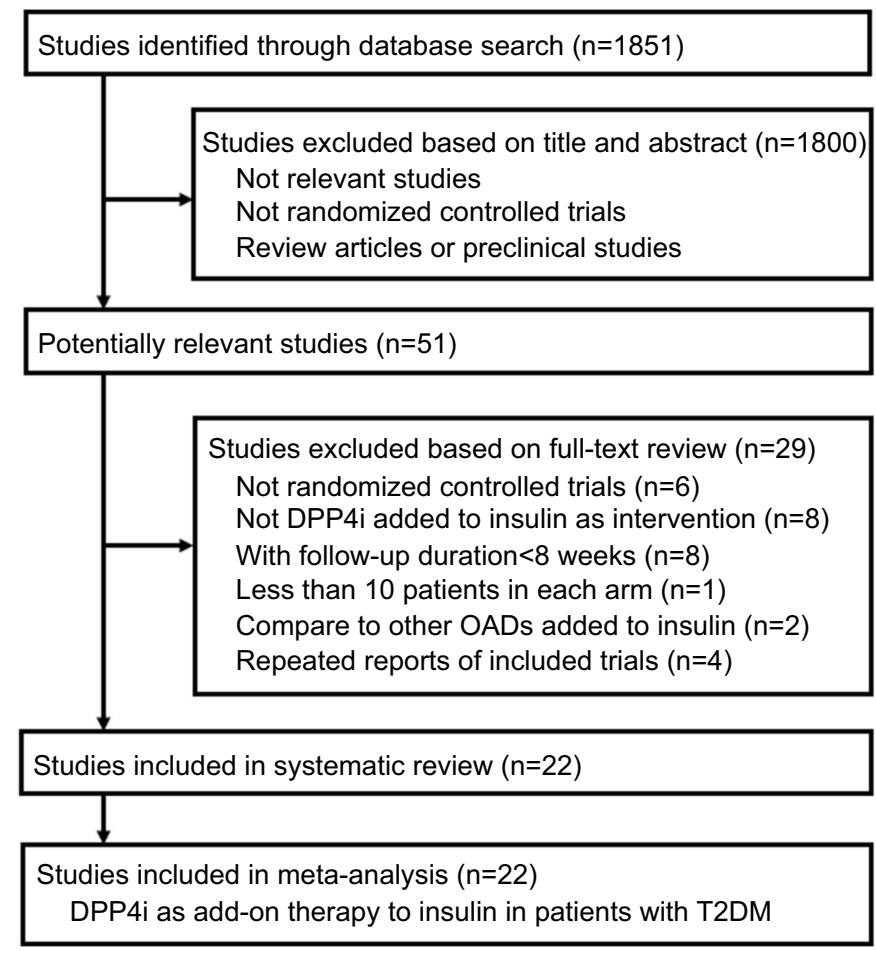

Figure I Summarized flowchart of literature search. 
two comparisons were considered for this study, which were included for analysis separately. The characteristics of the included RCTs are shown in Table 1. Briefly, the mean ages of the included patients varied from 51.0 to 69.1 years, and the baseline $\mathrm{HbA1c}$ ranged between $7.5 \%$ and $9.3 \%$. Sitagliptin was the most frequently used DPP4 inhibitors in these studies, followed by vildagliptin, alogliptin, saxagliptin, linagliptin, and teneligliptin. Most of the included studies included patients with various regimens of insulins, while two studies only included patients with basal insulin ${ }^{32,35}$ and another study $^{31}$ included patients with premix insulin (BIAsp30) only. The follow-up durations varied from 12 to 104 weeks. The doses of insulin were maintained stable in both arms in most of the included RCTs except for eight studies, in which the titration of insulin dose was allowed according to predefined algorithms. ${ }^{30,31,34-36,38,39,42}$ The summary of quality evaluation via a Cochrane's Risk of Bias Tool is shown in Table 2. Overall, the quality of the included studies was moderate. Fourteen studies were double-blinded and placebo-controlled RCTs. ${ }^{21-24,26-30,32,37,39,40,42}$ Methods of random sequence generation were reported in seven studies, ${ }^{23,26,28,36,38-40}$ while the strategies for allocation concealment were only reported in one study. ${ }^{40}$

\section{Effects of addition of DPP4 inhibitors to insulin on $\mathrm{HbAlc}$}

Meta-analysis with 22 studies showed that addition of DPP4 inhibitors to insulin was associated with significantly reduced $\mathrm{HbA} 1 \mathrm{c}$ as compared with controls (WMD: $-0.54 \%, 95 \% \mathrm{CI}$ : -0.66 to $-0.42, p<0.001$; Figure $2 \mathrm{~A}$ ) with significant heterogeneity $\left(\mathrm{I}^{2}=82 \%, \quad p\right.$ for Cochrane's $\mathrm{Q}$ test $\left.<0.001\right)$. Subsequent results of subgroup analyses showed that the effects of addition DPP4 inhibitors to insulin on HbAlc were consistent regardless of the study design characteristics, sample sizes of the RCTs, mean ages of the patients, followup durations of the studies, categories of the DPP4 inhibitors, or whether a stable or an up-titrated insulin dose was applied (Table 3). Interestingly, we found that the benefit of addition of DPP4 inhibitors to insulin on HbAlc was more remarkable in double-blinded, placebo-controlled RCTs, indicating the robustness of the findings. Moreover, trends could be observed that benefits of addition of DPP4 inhibitors to insulin on HbAlc may be more remarkable in short-term studies $(<24$ weeks) as compared with long-term studies ( $>24$ weeks, $p$ for subgroup difference $=0.06$ ), and in studies with stable insulin dose as compared with those with insulin dose titration ( $p$ for subgroup difference $=0.05$ ).

\section{Effects of addition of DPP4 inhibitors to insulin on FBG and PPG2h}

The pooled results showed that addition of DPP4 inhibitors to insulin was associated with significantly reduced FBG (WMD: $-0.47 \mathrm{mmol} / \mathrm{L}, 95 \% \mathrm{CI}:-0.71$ to -0.23 , $p<0.001 ; \mathrm{I}^{2}=58 \%$; Figure 2B) and PPG2h (WMD: -2.03 $\mathrm{mmol} / \mathrm{L}, 95 \%$ CI: -2.53 to $-1.54, p<0.001 ; \mathrm{I}^{2}=52 \%$; Figure 2C) as compared with controls.

\section{Effects of addition of DPP4 inhibitors to insulin on daily insulin dose, BW, and hypoglycemic events}

Addition of DPP4 inhibitors to insulin was found to be associated with a significantly reduced daily dose of insulin (WMD: $-2.73 \mathrm{U} / \mathrm{d}, 95 \% \mathrm{CI}:-3.96$ to $-1.51, p<0.001$; $\mathrm{I}^{2}=70 \%$; Figure $3 \mathrm{~A}$ ) as compared with controls. Moreover, addition of DPP4 inhibitors to insulin did not significantly affect BW (WMD: $0.02 \mathrm{Kg}, 95 \% \mathrm{CI}$ : -0.16 to 0.20 , $p=0.81 ; \mathrm{I}^{2}=32 \%$; Figure $3 \mathrm{~B}$ ) or risk of symptomatic hypoglycemia (RR: $0.92,95 \%$ CI: 0.78 to $1.10, p=0.37$; $\mathrm{I}^{2}=60 \%$; Figure $3 \mathrm{C}$ ) as compared with controls.

\section{Publication bias}

The funnel plots for the effects of DPP4 inhibitors as add-on therapy to insulin compared with controls on HbA1c, FBG, PPG2h, daily insulin dose, BW, and incidence of symptomatic hypoglycemic events are shown in Figure $4 \mathrm{~A}-\mathrm{F}$, which were all symmetrical on visual inspection symmetrical on visual inspection, suggesting no significant publication biases. These findings were further supported by the results of Egger's regression tests $(p=0.312,0.432,0.237,0.539,0.418$, and 0.602 , respectively).

\section{Discussion}

In this meta-analysis of RCTs, we found that addition of DPP4 inhibitors to insulin significantly improved the glycemic control in T2DM patients as compared with placebo/no treatment to insulin. Moreover, the benefits of DPP4 inhibitors as an addon therapy to insulin on glycemic control were consistent regardless of the study design, follow-up duration, and categories of DPP4 inhibitors used. In addition, the benefits of DPP4 inhibitors as an add-on therapy to insulin on glycemic control were observed not only in studies with stable insulin dose, but also in studies with insulin dose titration. Besides, the addition of DPP4 inhibitors to insulin significantly reduced daily insulin dose, without significant influences on $\mathrm{BW}$ or risk 


\begin{tabular}{|c|c|c|c|c|c|c|c|c|c|c|c|c|c|c|c|}
\hline \multicolumn{2}{|l|}{ 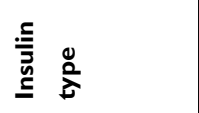 } & $\frac{\frac{0}{0}}{\frac{0}{5}}$ & $\frac{\frac{0}{0}}{\frac{\overline{2}}{\Sigma}}$ & $\frac{\frac{0}{0}}{\frac{0}{5}}$ & $\frac{\frac{0}{0}}{\frac{\overline{2}}{\Sigma}}$ & $\frac{\frac{0}{0}}{\frac{0}{5}}$ & $\frac{\frac{0}{0}}{\frac{0}{5}}$ & $\frac{\frac{0}{0}}{\frac{0}{5}}$ & $\frac{\frac{0}{2}}{\frac{2}{5}}$ & $\frac{\frac{0}{0}}{\frac{0}{5}}$ & $\frac{\frac{0}{2}}{\frac{.0}{5}}$ & 㤩 & 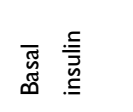 & $\frac{\frac{0}{0}}{\frac{0}{5}}$ & $\frac{\frac{0}{2}}{\frac{0}{5}}$ \\
\hline \multicolumn{2}{|l|}{ 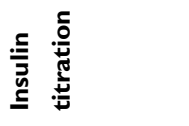 } & $z$ & $z$ & $z$ & $z$ & $z$ & $z$ & $z$ & $z$ & $z$ & $z$ & $\succ$ & $\succ$ & $z$ & $z$ \\
\hline 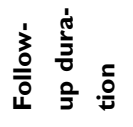 & 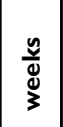 & $\stackrel{ \pm}{\sim}$ & $\stackrel{\sim}{\sim}$ & $\stackrel{\sim}{\sim}$ & $\stackrel{ \pm}{\sim}$ & $\stackrel{ \pm}{\sim}$ & $\stackrel{ \pm}{\Delta}$ & กี & $\underline{\circ}$ & $\stackrel{ \pm}{\sim}$ & $\simeq$ & $\stackrel{ \pm}{\sim}$ & $\stackrel{ \pm}{\sim}$ & $\simeq$ & $\simeq$ \\
\hline \multicolumn{2}{|l|}{ 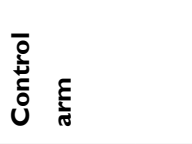 } & $\mp$ & $\mp$ & $\mp$ & $\mp$ & $\underset{0}{\mp}$ & 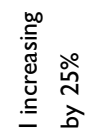 & $\mp$ & 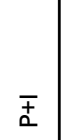 & $\mp$ & 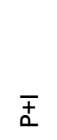 & 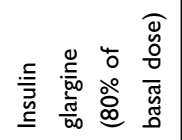 & 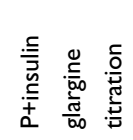 & $\Phi$ & - \\
\hline \multicolumn{2}{|l|}{ 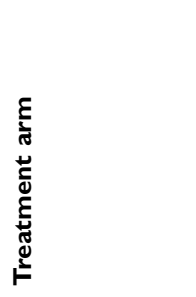 } & 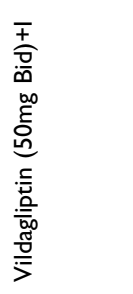 & 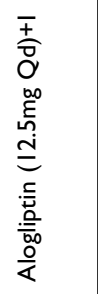 & 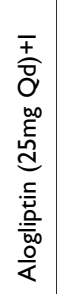 & 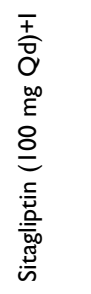 & 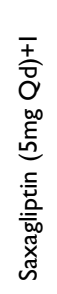 & 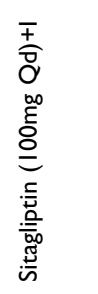 & 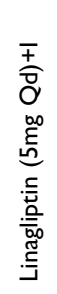 & 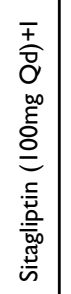 & 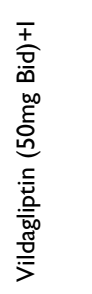 & 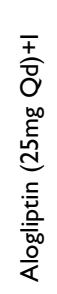 & 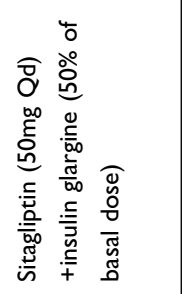 & 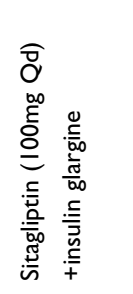 & 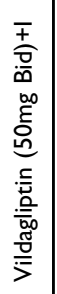 & 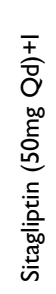 \\
\hline 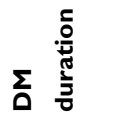 & 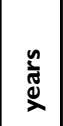 & $\stackrel{f}{\dot{I}}$ & $\overline{\underline{I}}$ & $\overline{\underline{m}}$ & $\stackrel{\stackrel{\sim}{\underline{I}}}{ }$ & $\simeq$ & 官 & $\mathbb{z}$ & $\overline{\dot{I}}$ & $\overline{\underline{m}}$ & $\underset{\dot{\dot{x}}}{\dot{q}}$ & $\mathbb{Z}$ & $\stackrel{\stackrel{m}{m}}{\underline{m}}$ & $\stackrel{a}{\dot{\mathcal{I}}}$ & $\stackrel{\circ}{\stackrel{0}{\Sigma}}$ \\
\hline 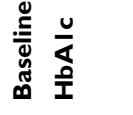 & ஃ。 & $\stackrel{+}{\infty}$ & $\stackrel{m}{\sigma}$ & $\stackrel{m}{a}$ & $\widehat{\infty}$ & $\hat{\infty}$ & $\stackrel{\sim}{\sigma}$ & $\underset{\infty}{m}$ & $\stackrel{a}{\infty}$ & 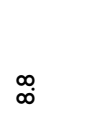 & $\underset{\infty}{\Delta}$ & $\stackrel{\llcorner}{\sim}$ & $\widehat{\infty}$ & $\bar{\infty}$ & ${\underset{\infty}{\infty}}_{\infty}^{m}$ \\
\hline$\overline{\Sigma_{\infty}}$ & 䓂 & $\bar{m}$ & $\underset{\text { ¿্ల }}{ }$ & 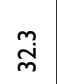 & $\bar{m}$ & $\underset{\sim}{\sim}$ & 㞫 & $\bar{m}$ & นึ่ & సे & $\stackrel{m}{\stackrel{m}{\sim}}$ & $\underset{\tilde{\sim}}{\stackrel{\sigma}{n}}$ & $\overline{\dot{m}}$ & 虫 & $\underset{\sim}{\sim}$ \\
\hline$\frac{\frac{0}{\pi}}{\Sigma}$ & $\circ$ & $\frac{\text { ti. }}{\text { in }}$ & $\stackrel{\infty}{\dddot{F}}$ & $\stackrel{\circ}{\infty}$ & $\overline{i n}$ & $\frac{m}{\sigma}$ & $\stackrel{m}{\text { Ch }}$ & $\overline{\mathrm{r}}$ & $\hat{\infty}$ & $\bar{i}$ & 疋 & $\frac{\pi}{\sigma}$ & $\underset{\stackrel{m}{+}}{\stackrel{f}{r}}$ & $\stackrel{\sim}{\wedge}$ & $\frac{9}{0}$ \\
\hline 离 & 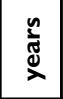 & กั่ & ભm & $\hat{n}$ & $\hat{i}$ & $\stackrel{m}{i}$ & ก้ & $\bar{o}$ & $\frac{1}{0}$ & กั & $\widehat{i}$ & 華 & 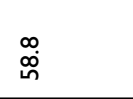 & $\stackrel{m}{\sigma^{n}}$ & $\bar{s}$ \\
\hline 告 & & ঐ̊ & $\stackrel{\circ}{\circ}$ & $\stackrel{ \pm}{\sigma}$ & $\overline{\mathrm{g}}$ & 㤐 & $\stackrel{q}{q}$ & $\overline{\stackrel{\Xi}{9}}$ & $\stackrel{\circ}{\sim}$ & $\underset{f}{q}$ & $\stackrel{\Omega}{\underline{\Omega}}$ & $\Varangle$ & : & $\stackrel{\circ}{\stackrel{\circ}{2}}$ & $\bar{N}$ \\
\hline نे & & 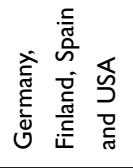 & 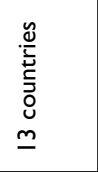 & 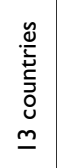 & 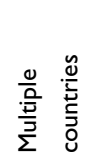 & 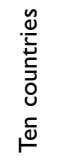 & $\begin{array}{l}\stackrel{\Xi}{0} \\
\stackrel{0}{0}\end{array}$ & 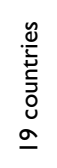 & 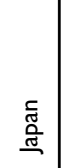 & 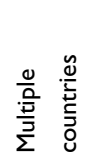 & 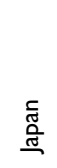 & 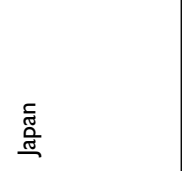 & 爻 & 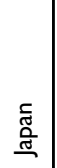 & 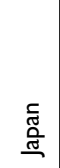 \\
\hline 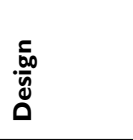 & & $\begin{array}{l}u \\
0 \\
\tilde{a}^{\prime} \\
\check{x}\end{array}$ & $\begin{array}{l}u \\
0 \\
\infty \\
\alpha \\
\propto\end{array}$ & $\begin{array}{l}u \\
a \\
\infty \\
0 \\
\propto\end{array}$ & $\begin{array}{l}u \\
0 \\
0 \\
0 \\
\propto\end{array}$ & $\begin{array}{l}u \\
\vdots \\
\infty \\
0 \\
\propto\end{array}$ & $\begin{array}{l}u \\
\vdots \\
\propto\end{array}$ & $\begin{array}{l}u \\
0 \\
\infty \\
0 \\
\alpha\end{array}$ & $\begin{array}{l}u \\
0 \\
\infty \\
a \\
\alpha\end{array}$ & $\begin{array}{l}u \\
\alpha \\
\infty \\
a \\
\alpha\end{array}$ & $\begin{array}{l}\text { un } \\
\infty \\
0 \\
\alpha\end{array}$ & $\propto$ & $\begin{array}{l}u \\
0 \\
\infty \\
0 \\
\propto\end{array}$ & 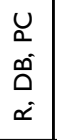 & $\propto$ \\
\hline 斉 & & 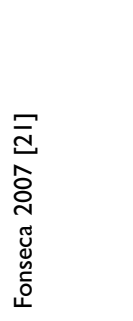 & 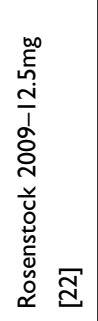 & 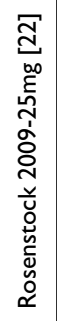 & 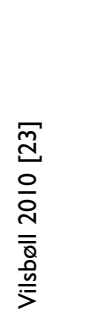 & 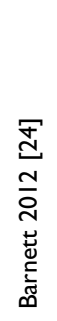 & 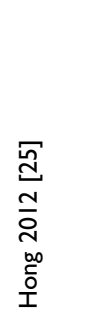 & 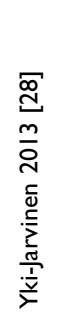 & 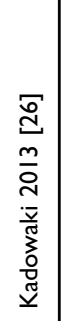 & 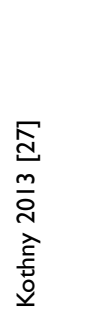 & 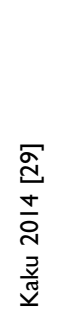 & 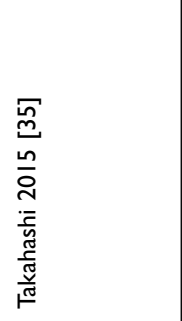 & 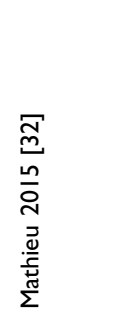 & 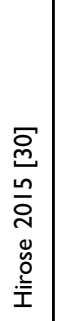 & 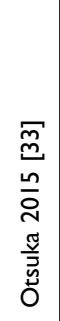 \\
\hline
\end{tabular}




\begin{tabular}{|c|c|c|c|c|c|c|c|c|c|c|c|}
\hline \multicolumn{2}{|l|}{ 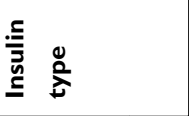 } & $\begin{array}{l}0 \\
\frac{0}{0} \\
\frac{1}{\infty} \\
\frac{1}{\infty}\end{array}$ & $\frac{\frac{0}{2}}{\frac{0}{5}}$ & $\frac{\frac{0}{2}}{\frac{0}{5}}$ & $\frac{\frac{0}{2}}{\frac{0}{5}}$ & $\frac{\frac{0}{2}}{\frac{0}{\overline{2}}}$ & $\frac{\frac{0}{20}}{\frac{0}{5}}$ & $\frac{\frac{0}{2}}{\frac{2}{5}}$ & $\frac{\frac{0}{2}}{\frac{0}{5}}$ & \begin{tabular}{|l|}
$\frac{0}{2}$ \\
$\frac{2}{5}$ \\
$\frac{5}{\Sigma}$
\end{tabular} & \\
\hline \multicolumn{2}{|l|}{ 皇 } & $\succ$ & $\succ$ & $\succ$ & $z$ & $z$ & $\succ$ & $\succ$ & $z$ & $\succ$ & $\underset{\stackrel{\dot{\omega}}{\grave{\nu}}}{\stackrel{\dot{\nu}}{\nu}}$ \\
\hline 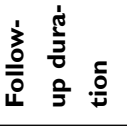 & \begin{tabular}{|l|}
$\frac{n}{\tilde{u}}$ \\
3 \\
3
\end{tabular} & $\stackrel{ \pm}{\sim}$ & $\stackrel{ \pm}{\sim}$ & ț & $\stackrel{ \pm}{\sim}$ & $\stackrel{t}{\underline{0}}$ & $\underline{\varrho}$ & 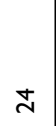 & $\underline{\varrho}$ & $\underline{0}$ & \begin{tabular}{l}
$z$ \\
$\dot{p}$ \\
\multirow{j}{*}{}
\end{tabular} \\
\hline \multicolumn{2}{|l|}{ 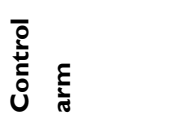 } & $\begin{array}{l}\text { o } \\
\text { 产 } \\
\frac{1}{\infty}\end{array}$ & - & - & $\mp$ & - & 品 & $\mp$ & $\mp$ & $\mp$ & $\begin{array}{l}\overline{\overline{\bar{z}}} \\
\stackrel{\underline{\underline{g}}}{\overline{\bar{g}}}\end{array}$ \\
\hline \multicolumn{2}{|l|}{ 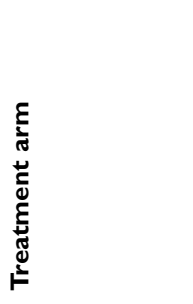 } & 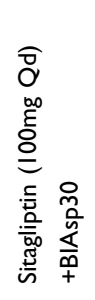 & 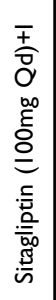 & 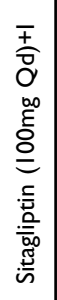 & 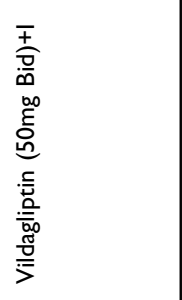 & 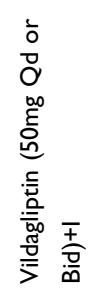 & 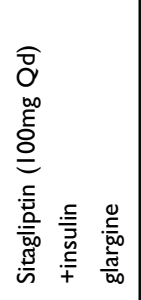 & 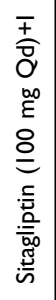 & 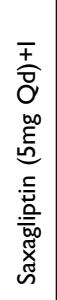 & 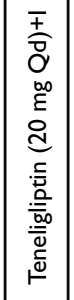 & 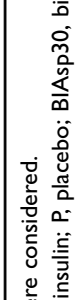 \\
\hline 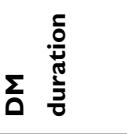 & 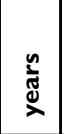 & $\overleftarrow{z}$ & $\stackrel{\llcorner}{\sigma}$ & $\stackrel{m}{=}$ & $\stackrel{m}{=}$ & $\underline{\infty}$ & 0 & $\stackrel{\stackrel{ }{=}}{=}$ & $\stackrel{\infty}{\stackrel{\infty}{\underline{\rho}}}$ & 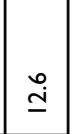 & 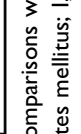 \\
\hline 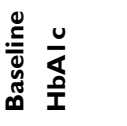 & ஃ & $\stackrel{+}{\infty}$ & $\stackrel{\sigma}{r}$ & $\bar{\infty}$ & $\widehat{\infty}$ & $\stackrel{a}{\wedge}$ & $\underset{\infty}{\sim}$ & $\widehat{\infty}$ & $\underset{\infty}{m}$ & $\hat{\infty}$ & 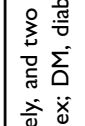 \\
\hline $\bar{\Sigma}$ & $\underline{\tilde{\xi}}$ & 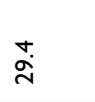 & $\hat{\mathrm{d}}$ & $\stackrel{\sim}{\sim}$ & $\bar{i}$ & $\stackrel{m}{\sim}$ & 苛 & $\stackrel{\sim}{ }$ & $\overline{\dot{\sim}}$ & $\begin{array}{l}\dot{d} \\
\dot{d}\end{array}$ & 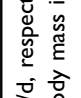 \\
\hline$\frac{0}{\bar{\Sigma}}$ & $\circ$ & $\overline{\mathrm{i}}$ & t. & 圆 & $\underset{\tilde{q}}{\tilde{f}}$ & $\tilde{0}$ & $\stackrel{0}{\operatorname{nn}}$ & $\stackrel{m}{n}$ & $\bar{\sigma}$ & $\stackrel{\text { ஜ̊ }}{\stackrel{2}{*}}$ & 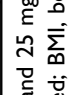 \\
\hline 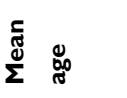 & 足 & 䓟 & $\stackrel{\circ}{\circ}$ & $\hat{\tilde{j}}$ & $\bar{\infty}$ & $\bar{\sigma}$ & $\bar{n}$ & $\hat{i n}$ & 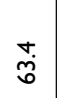 & $\begin{array}{c}\stackrel{n}{\infty} \\
\infty \\
\sim\end{array}$ & 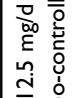 \\
\hline 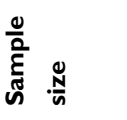 & & $\stackrel{\circ}{\stackrel{\rho}{m}}$ & in & $\underset{\sim}{\sim}$ & $\stackrel{\tilde{N}}{ }$ & 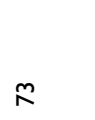 & $\tilde{0}$ & $\hat{f}$ & $\tilde{\widetilde{\sim}}$ & 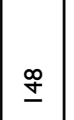 & 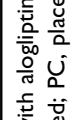 \\
\hline نे & & 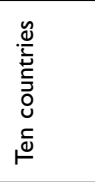 & $\begin{array}{l}\text { 疍 } \\
\text { 胥 }\end{array}$ & $\begin{array}{l}\text { 胥 } \\
\text { 蒠 }\end{array}$ & 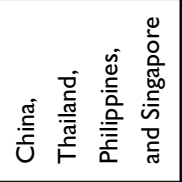 & $\begin{array}{l}\text { 대 } \\
\text { 尊 }\end{array}$ & 疍 & 趇 & 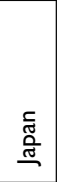 & 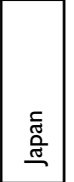 & 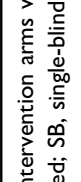 \\
\hline $\begin{array}{l}\frac{5}{50} \\
\overline{\bar{D}} \\
\Delta\end{array}$ & & $\propto$ & $\propto$ & $\begin{array}{l}\mathscr{n} \\
\check{c}\end{array}$ & $\begin{array}{l}u \\
\alpha \\
\Delta \\
\alpha \\
\alpha\end{array}$ & $\propto$ & $\propto$ & $\begin{array}{l}u \\
\alpha \\
\infty \\
0 \\
\alpha\end{array}$ & $\begin{array}{l}u \\
\alpha \\
\infty \\
\alpha \\
\propto ்\end{array}$ & $\begin{array}{l}u \\
\alpha \\
\tilde{a} \\
a \\
\alpha\end{array}$ & 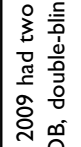 \\
\hline 害 & & 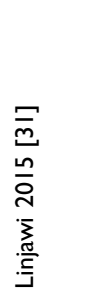 & 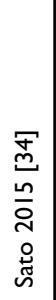 & 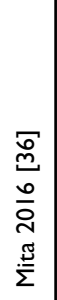 & 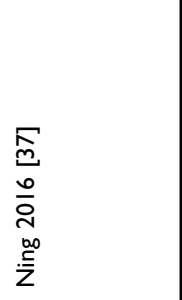 & 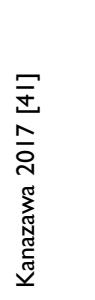 & $\begin{array}{l}\infty \\
\stackrel{\infty}{0} \\
\hat{\bar{i}} \\
o \\
\stackrel{\Xi}{0}\end{array}$ & 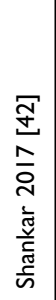 & 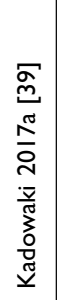 & 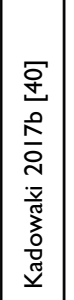 & 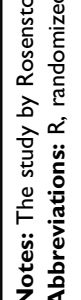 \\
\hline
\end{tabular}




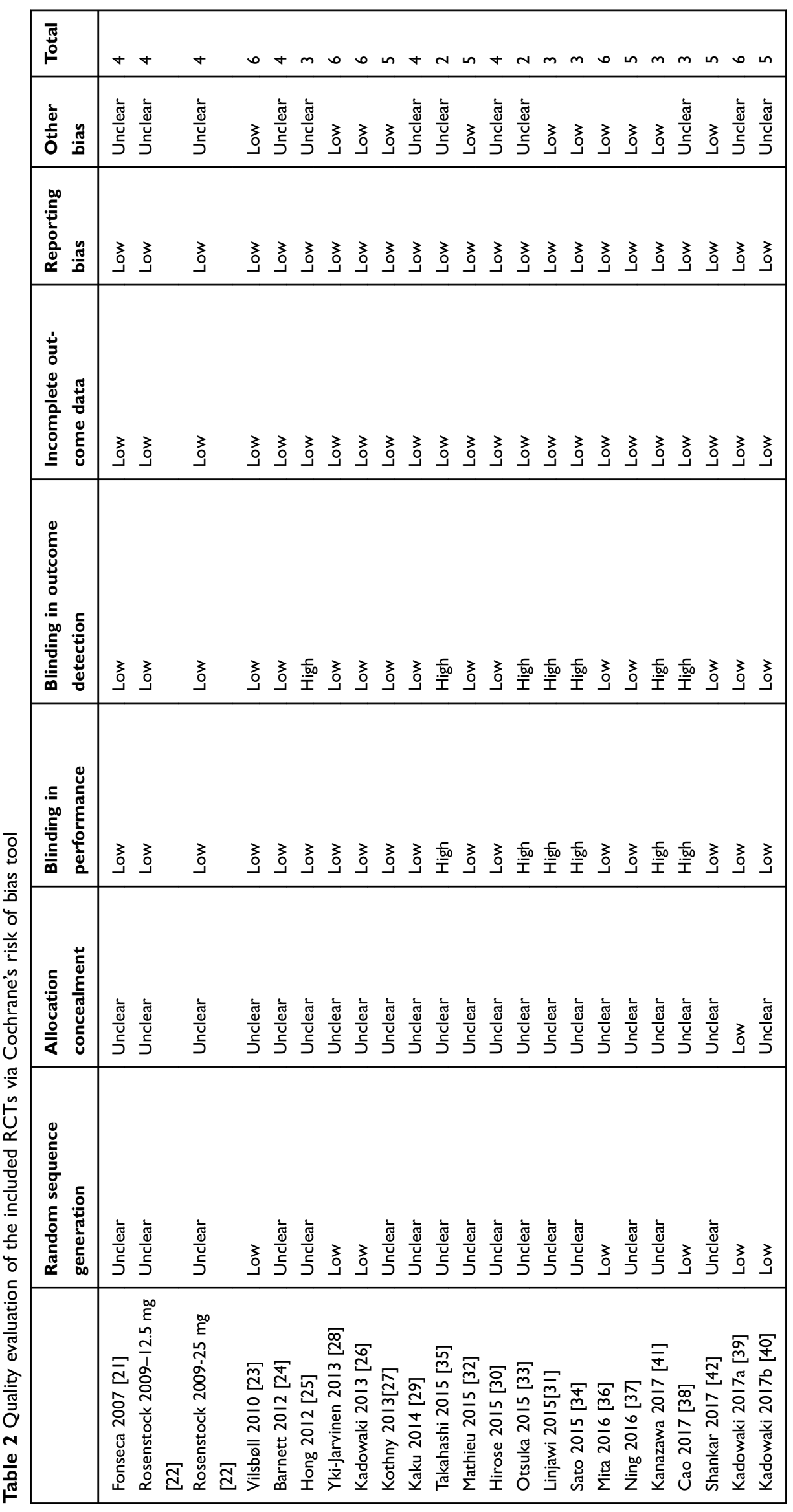



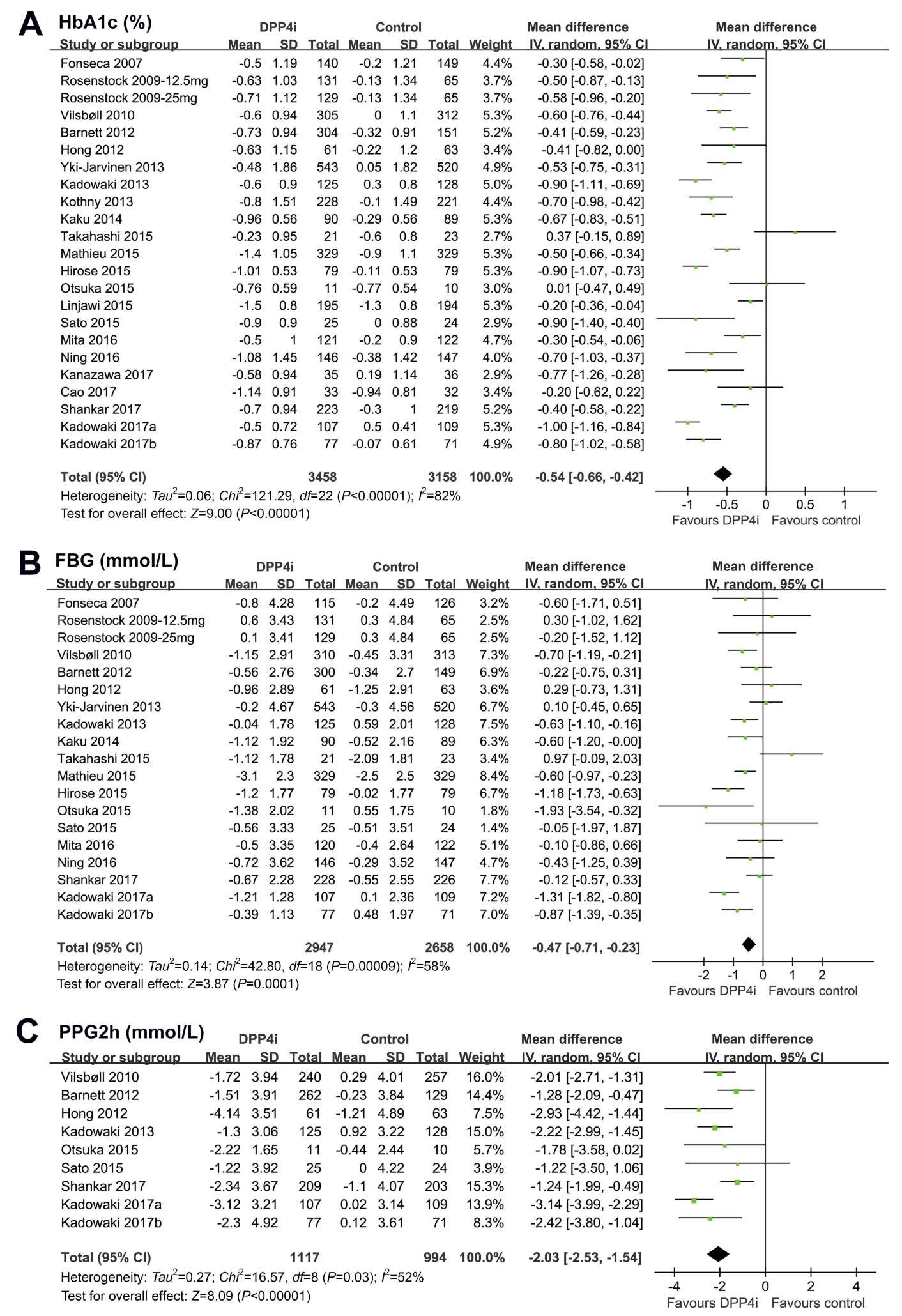

Figure 2 Forest plots for the meta-analyses of addition of the dipeptidyl peptidase-4 inhibitors to insulin on glycemic control in patients with type 2 diabetes mellitus. (A) $\mathrm{HbAlc}(\%)$; (B) fasting blood glucose ( $\mathrm{mmol} / \mathrm{L})$; and (C) postprandial glucose at $2 \mathrm{hrs}$.

of hypoglycemia. These results suggested that DPP4 inhibitors as an add-on therapy to insulin improved glycemic control without further increasing the risk of weight gain and hypoglycemia in patients with T2DM. 


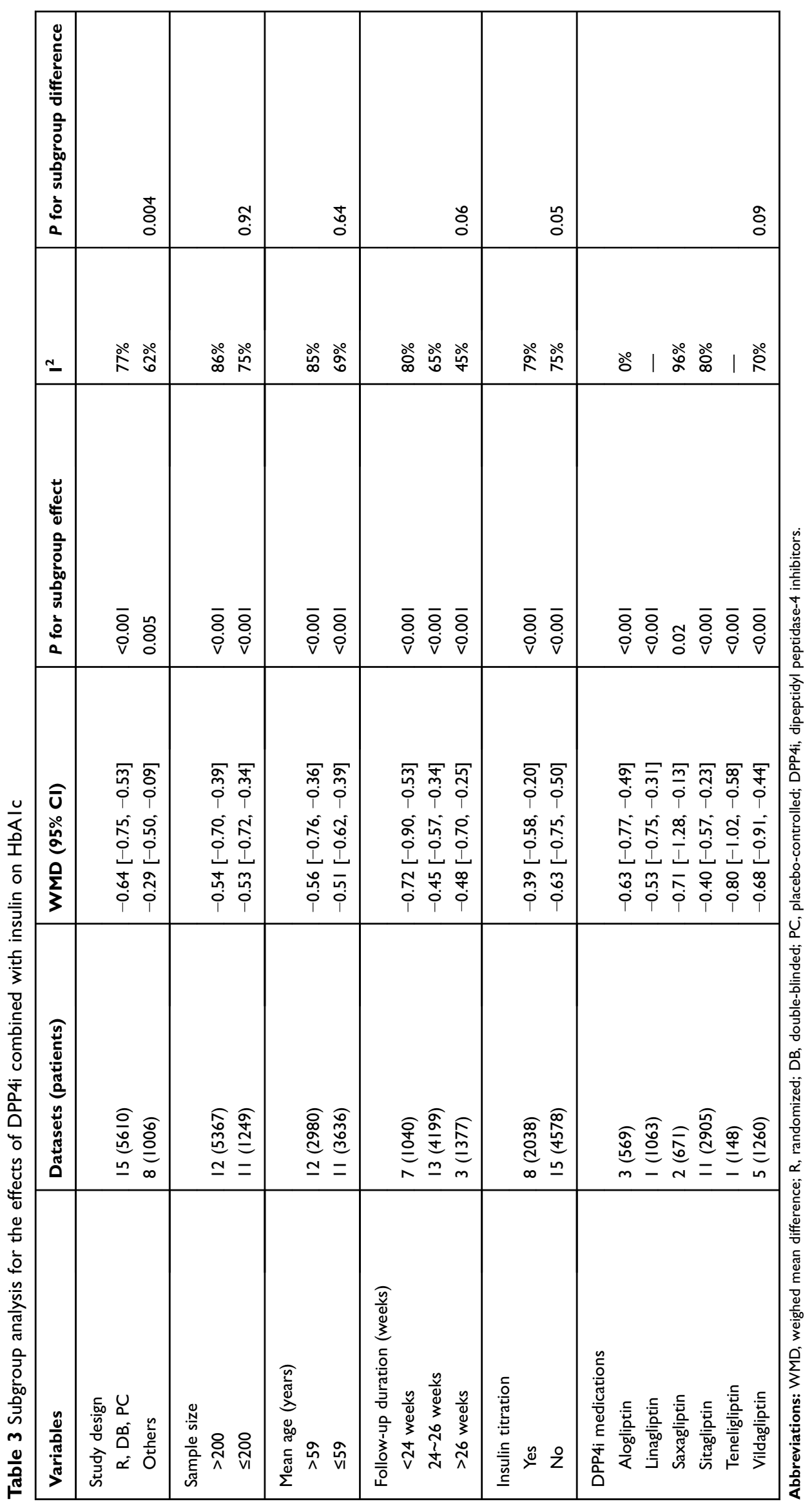




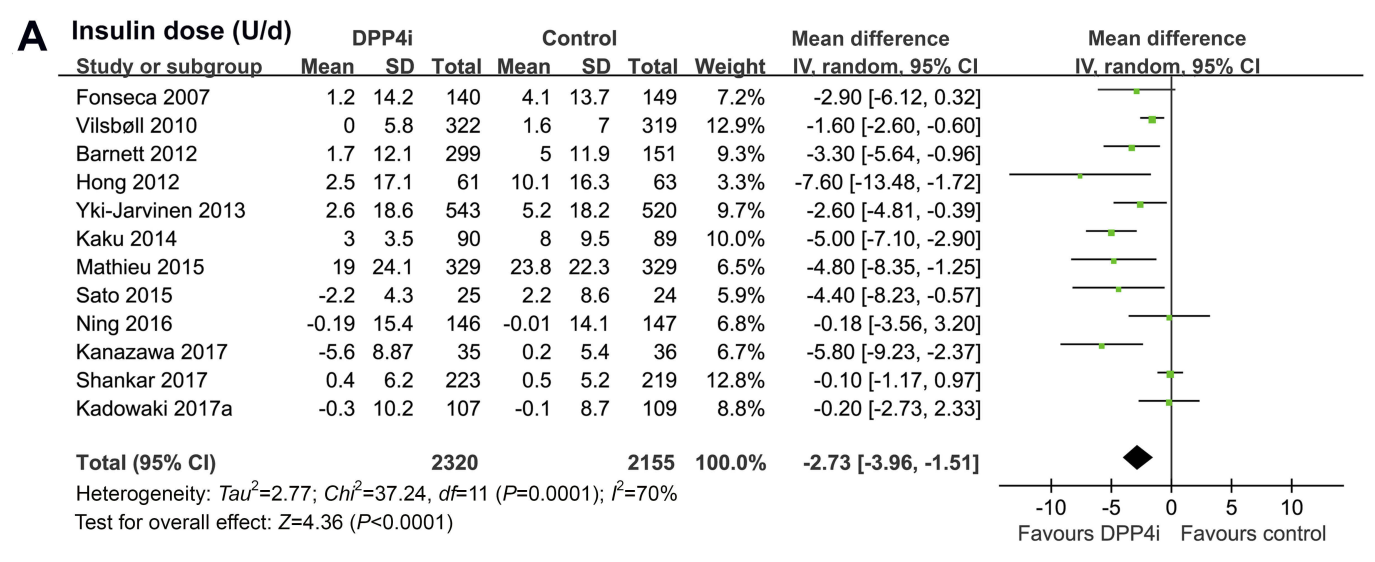

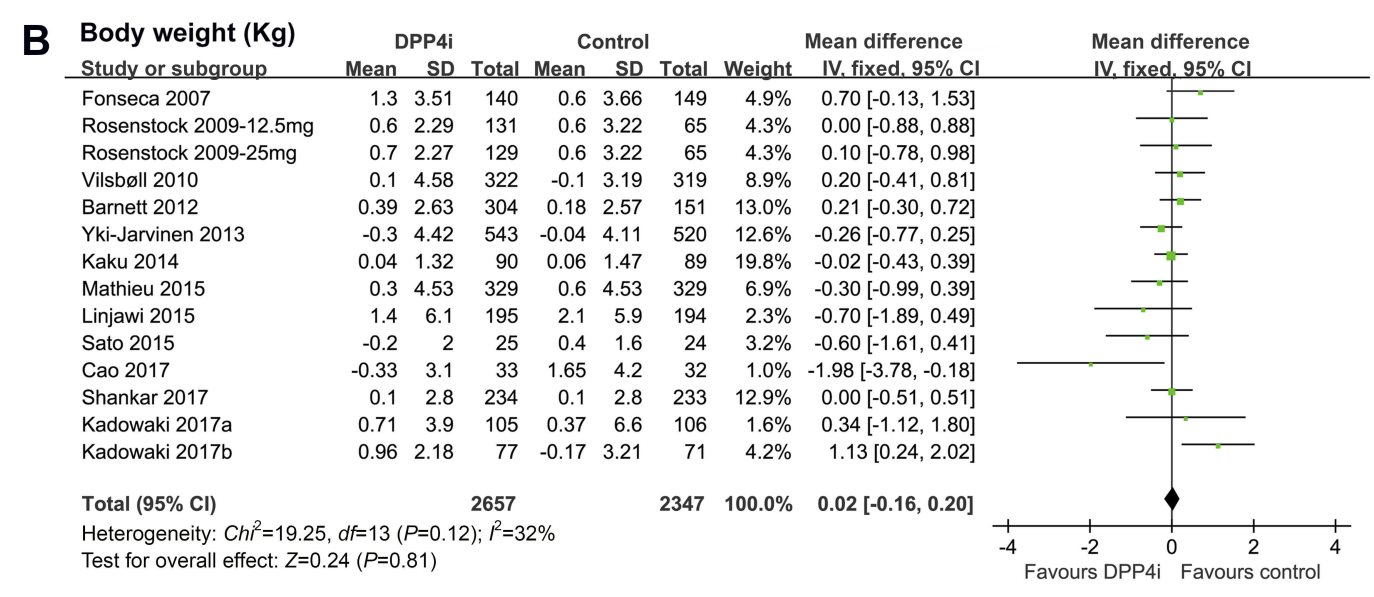

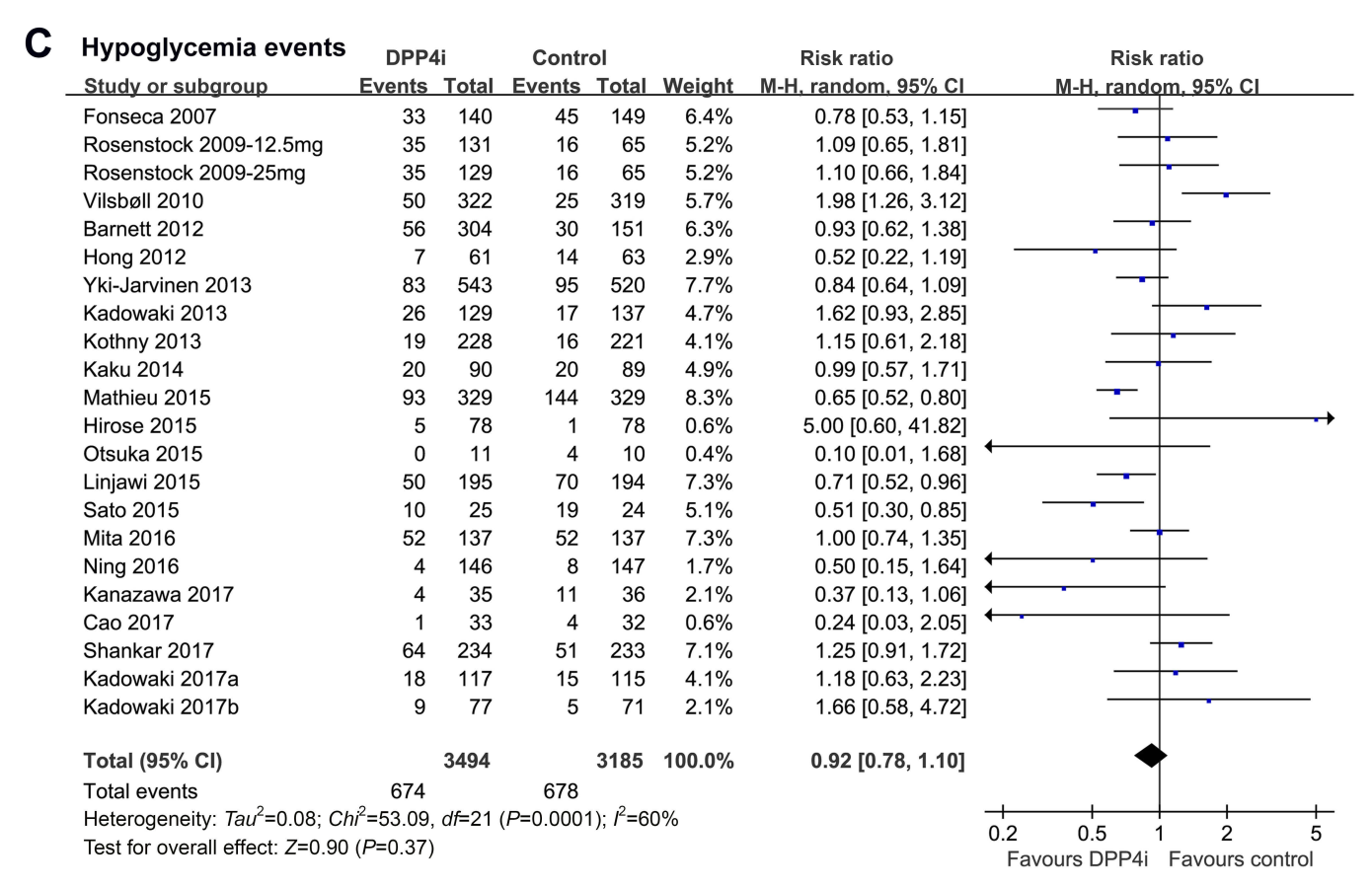

Figure 3 Forest plots for the meta-analyses of addition of the dipeptidyl peptidase-4 inhibitors to insulin on daily insulin dose and safety outcomes. (A) daily insulin dose (U/d); (B) body weight $(\mathrm{kg})$; and $(\mathbf{C})$ incidence of systematic hypoglycemia.

Previously, two meta-analyses performed by Chen et $\mathrm{al}^{43}$ and Kim et $\mathrm{al}^{44}$ have been published concerning the efficacy of DPP4 inhibitors as add-on therapy to insulin. Our study is different from the previous two meta-analyses in the 

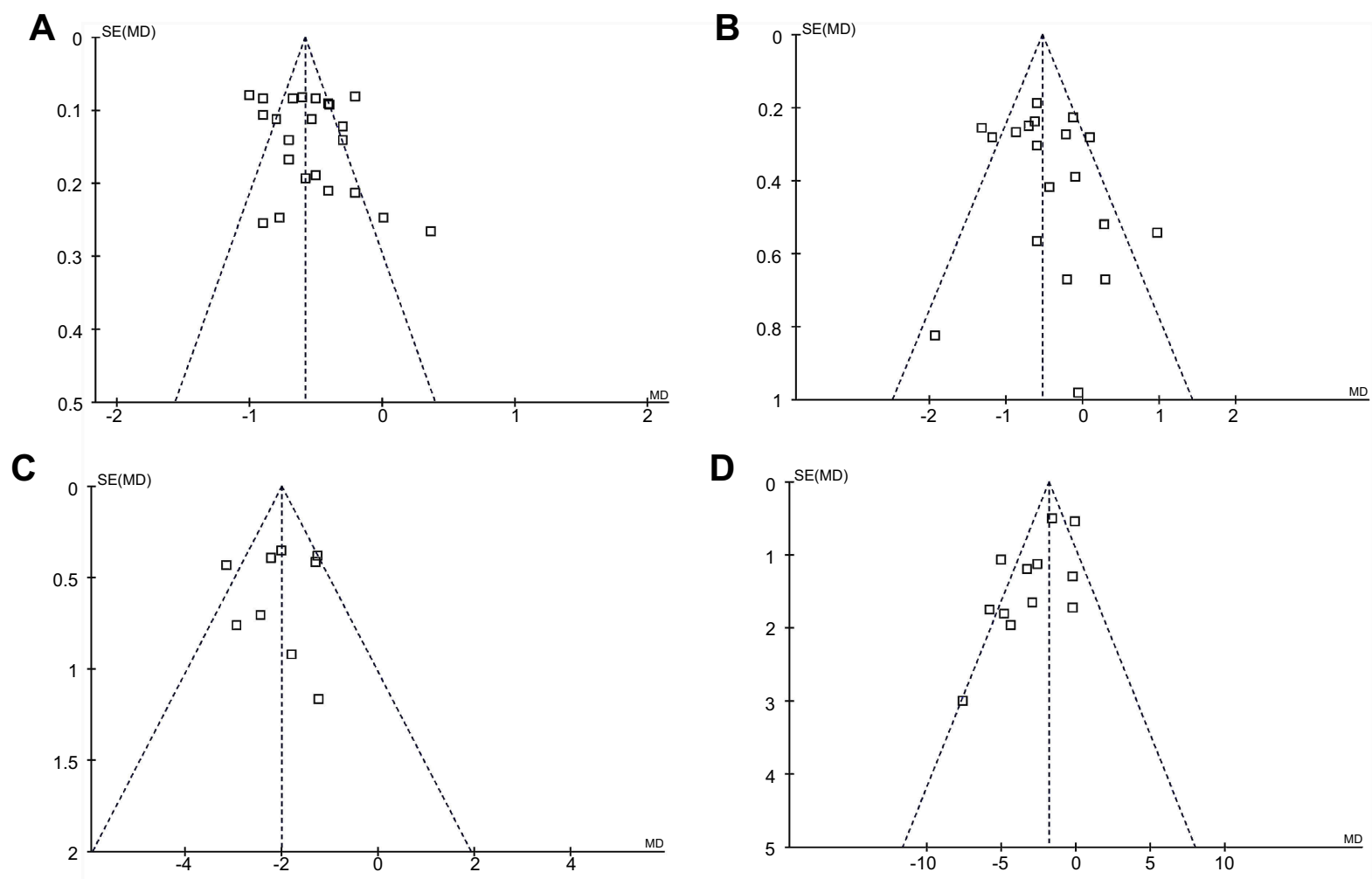

D

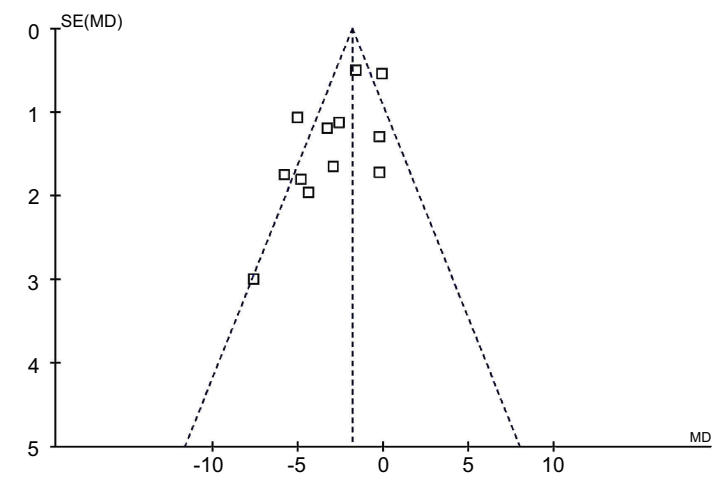

E

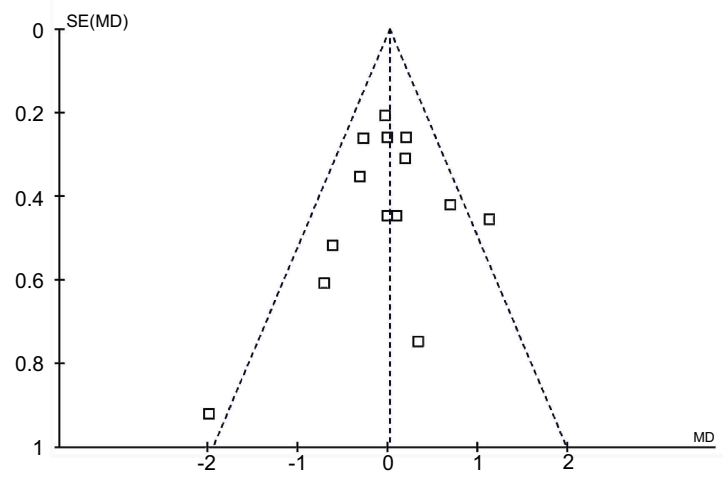

F

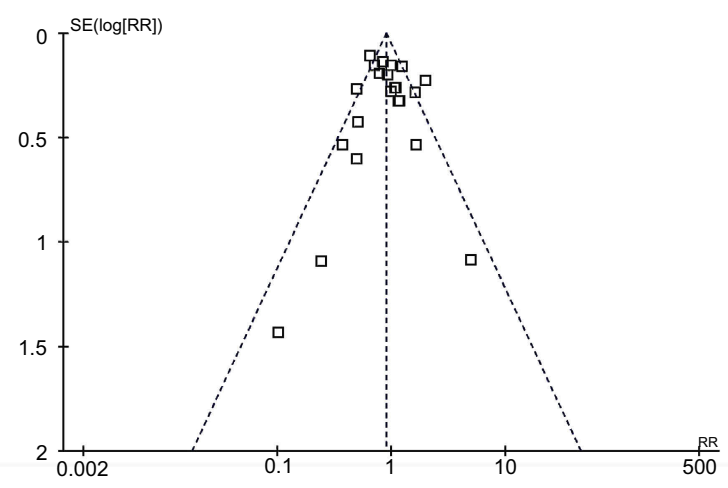

Figure 4 Funnel plots for the meta-analyses. (A) HbAlc (\%); (B) fasting blood glucose (mmol/L); (C) postprandial glucose at 2 hrs; (D) daily insulin dose (U/d); (E) body weight $(\mathrm{kg})$; and $(\mathbf{F})$ incidence of systematic hypoglycemia.

following aspects. Firstly, the meta-analysis by Chen et al included RCTs comparing the effect between DPP4 inhibitors and placebo/no treatment/active OADs as add-on therapies to insulin. ${ }^{43}$ The various regimens of controls in this meta-analysis may confound the results. In the meta-analysis by Kim et al, the authors focused on studies with stable insulin dose, while the potential glycemic benefits of addition of DPP4 inhibitors to insulin with adjustable dose therefore cannot be confirmed. ${ }^{44}$ However, in our meta-analysis, we included all RCTs comparing the effect of DPP4 inhibitors and placebo/no treatment controls as add-on therapies to insulin in T2DM patients, and the dose of insulin in the included RCTs could be stable or adjustable (titration) during the study periods. Secondly, the numbers of included RCTs (seven in meta-analysis of Chen et al, nine in meta-analysis of Kim et al, and 22 in our meta-analysis) and the overall patients (3,384 in meta-analysis of Chen et al, 4,464 in metaanalysis of Kim et al, and 6,957 in our meta-analysis) were much larger in our study than the previous ones, which makes our study with larger statistical power to show a significantly improved FBG and PPG2h in DPP4 inhibitor groups. Thirdly, the large scale of the current meta-analysis allowed us to perform subgroup analysis for the influence of the combined treatment with DPP4 inhibitors and insulin on HbA1c. Generally, the results of our meta-analysis are consistent with the previous two meta-analyses, ${ }^{4,44}$ which 
showed that addition of DPP4 inhibitors to insulin improved glycemic control in T2DM patients. Moreover, results of our subgroup analyses showed that the benefits of DPP4 inhibitors as an add-on therapy to insulin on glycemic control were consistent regardless of the study design, follow-up duration, and categories of DPP4 inhibitors used. Finally, we found that benefits of DPP4 inhibitors as an add-on therapy to insulin on glycemic control were observed not only in studies with stable insulin dose, but also in studies with insulin dose titration. These results implied that in T2DM patients that are inadequately controlled by insulin, adding DPP4 inhibitors to insulin may be superior in glycemic control as compared with the up-titration of the insulin dose.

The synergetic effect of the addition of DPP4 inhibitors to insulin therapy on glycemic control may be explained by the potential insulin-independent hypoglycemic effect of DPP4 inhibitors. The DPP4 inhibitors prevent the degradation of gastrointestinal incretins including GLP-1and GIPs, thereby improving glycemic control via various mechanisms besides stimulation of insulin secretion, such as glucagon suppression. ${ }^{49}$ The complementary actions of DPP4 inhibitors to insulin therapy may be the fundamental reasons for the benefits of combined therapy on glycemic control. This is also reflected by one of the findings from our meta-analysis which showed a significantly reduced daily insulin dose in patients receiving combined therapy with DPP4 inhibitors and insulin. Moreover, our meta-analysis showed that addition of DPP4 inhibitors to insulin did not the risks of BW gain and hypoglycemia. This is not surprising since DPP4 inhibitors are confirmed to have no significant influence on BW and with low risk of hypoglycemic events. ${ }^{19}$ Additionally, patients from the combined therapy group had lower daily insulin dose than controls as evidenced by the results of our meta-analysis, which may also be a potential reason that the combined treatment did not significantly increase the risk of adverse events such as weight gain and hypoglycemia.

Our study has limitations. Firstly, our study is a studylevel-based meta-analysis. Results of subgroup analysis according to the study or patient characteristics (such as mean ages, and follow-up duration) should be interpreted with caution since we did perform the stratified analyses based on the individual patient data. Secondly, significant heterogeneity remains in some outcomes of our meta-analysis, and differences in the regimens of insulin treatment of the included RCTs may contribute to the heterogeneity. However, subgroup analyses according to the regimens of insulin treatment could not be performed since most of the RCTs included patients with mixed insulin regimens, while stratified data were rarely available. In one of the included RCTs, addition of sitagliptin various regimens of insulin, including premixed, immediate-acting, and long-acting insulin showed similar efficacies on $\mathrm{HbA} 1 \mathrm{c}$ reduction in T2DM patients. ${ }^{23}$ Similarly, a recent post-hoc analysis of two clinical trials showed that sitagliptin in combination with premixed insulin achieved better glycemic control than premixed insulin alone. ${ }^{50}$ Therefore, whether addition of DPP4 inhibitors to different regimens of insulin treatment confers similar benefits remains to be determined. Thirdly, the comparative efficacies between DPP4 inhibitors and other active OADs as add-on therapies to insulin cannot be determined based on our results, and direct comparative RCTs are lacking. Previous meta-analyses based on indirect comparisons showed that DPP4 inhibitors may be inferior to sodium glucose cotransporter 2 inhibitors, ${ }^{51}$ but similar to thiazolidinedione and GLP-1 receptor agonists in glycemic control as add-on therapies to insulin in T2DM patients. ${ }^{52}$ However, these results should be validated in head-to-head RCTs. Fourthly, whether addition of DPP4 inhibitors to insulin improves the clinical outcome should be evaluated in future studies.

In conclusion, results of our meta-analysis showed that addition of DPP4 inhibitors to insulin significantly improved the glycemic control in T2DM patients without further increasing the risk of weight gain and hypoglycemia. The benefits of DPP4 inhibitors as add-on therapy on glycemic control were independent of study design, follow-up duration, categories of DPP4 inhibitors used, and using of fixed/adjustable insulin doses. The DPP4 inhibitors as an add-on therapy to insulin should be considered in T2DM patients in clinical practice.

\section{Disclosure}

The authors report no conflicts of interest in this work.

\section{References}

1. Lascar N, Brown J, Pattison H, Barnett AH, Bailey CJ, Bellary S. Type 2 diabetes in adolescents and young adults. Lancet Diabetes Endocrinol. 2018;6(1):69-80. doi:10.1016/S2213-8587(17)30186-9

2. Yang W, Lu J, Weng J, et al. Prevalence of diabetes among men and women in China. $N$ Engl $J$ Med. 2010;362(12):1090-1101. doi:10.1056/NEJMoa0908292

3. Worldwide trends in diabetes since. 1980: a pooled analysis of 751 population-based studies with 4.4 million participants. Lancet. 2016;387(10027):1513-1530. doi:10.1016/S0140-6736(16)00618-8

4. Czech MP. Insulin action and resistance in obesity and type 2 diabetes. Nat Med. 2017;23(7):804-814. doi:10.1038/nm.4350

5. Valaiyapathi B, Gower B, Ashraf AP. Pathophysiology of type 2 diabetes in children and adolescents. Curr Diabetes Rev. 2018. doi:10.2174/1573399814666180608074510 
6. Chamberlain JJ, Herman WH, Leal S, et al. Pharmacologic therapy for type 2 diabetes: synopsis of the 2017 American Diabetes Association Standards of Medical Care in Diabetes. Ann Intern Med. 2017;166 (8):572-578. doi:10.7326/M16-2937

7. Weng J, Ji L, Jia W, et al. Standards of care for type 2 diabetes in China. Diabetes Metab Res Rev. 2016;32(5):442-458. doi:10.1002/dmrr.2827

8. Raccah D, Huet D, Dib A, et al. Review of basal-plus insulin regimen options for simpler insulin intensification in people with Type 2 diabetes mellitus. Diabet Med. 2017;34(9):1193-1204. doi:10.1111/dme.13390

9. Giugliano D, Sieradzki J, Stefanski A, Gentilella R. Personalized intensification of insulin therapy in type 2 diabetes - does a basalbolus regimen suit all patients? Curr Med Res Opin. 2016;32 (8):1425-1434. doi:10.1080/03007995.2016.1181051

10. Wang C, Mamza J, Idris I. Biphasic vs basal bolus insulin regimen in Type 2 diabetes: a systematic review and meta-analysis of randomized controlled trials. Diabet Med. 2015;32(5):585-594. doi:10.1111/dme.12694

11. Goh SY, Hussein Z, Rudijanto A. Review of insulin-associated hypoglycemia and its impact on the management of diabetes in Southeast Asian countries. J Diabetes Investig. 2017;8(5):635-645. doi:10.1111/jdi.12647

12. Brown A, Guess N, Dornhorst A, Taheri S, Frost G. Insulin-associated weight gain in obese type 2 diabetes mellitus patients: what can be done? Diabetes Obes Metab. 2017;19(12):1655-1668. doi:10.1111/dom.13009

13. Stein SA, Lamos EM, Davis SN. A review of the efficacy and safety of oral antidiabetic drugs. Expert Opin Drug Saf. 2013;12(2):153175. doi:10.1517/14740338.2013.752813

14. Daniels MA, Kan C, Willmes DM, et al. Pharmacogenomics in type 2 diabetes: oral antidiabetic drugs. Pharmacogenomics J. 2016;16 (5):399-410. doi:10.1038/tpj.2016.54

15. Del Prato S, Bianchi C, Marchetti P. beta-cell function and anti-diabetic pharmacotherapy. Diabetes Metab Res Rev. 2007;23(7):518-527. doi:10.1002/dmrr.770

16. Waldrop G, Zhong J, Peters M, Rajagopalan S. Incretin-based therapy for diabetes: what a cardiologist needs to know. J Am Coll Cardiol. 2016;67(12):1488-1496. doi:10.1016/j.jacc.2015.12.058

17. Esposito K, Chiodini P, Maiorino MI, Bellastella G, Capuano A, Giugliano D. Glycaemic durability with dipeptidyl peptidase-4 inhibitors in type 2 diabetes: a systematic review and meta-analysis of long-term randomised controlled trials. BMJ Open. 2014;4(6) e005442. doi:10.1136/bmjopen-2014-005442

18. Drucker DJ, Nauck MA. The incretin system: glucagon-like peptide1 receptor agonists and dipeptidyl peptidase- 4 inhibitors in type 2 diabetes. Lancet. 2006;368(9548):1696-1705. doi:10.1016/S01406736(06)69705-5

19. Ling J, Cheng P, Ge L, et al. The efficacy and safety of dipeptidyl peptidase- 4 inhibitors for type 2 diabetes: a Bayesian network metaanalysis of 58 randomized controlled trials. Acta Diabetol. 2019;56 (3):249-272.

20. Ostergaard L, Frandsen CS, Madsbad S. Treatment potential of the GLP-1 receptor agonists in type 2 diabetes mellitus: a review. Expert Rev Clin Pharmacol. 2016;9(2):241-265. doi:10.1586/17512433.2016.1121808

21. Fonseca V, Schweizer A, Albrecht D, Baron MA, Chang I, Dejager S. Addition of vildagliptin to insulin improves glycaemic control in type 2 diabetes. Diabetologia. 2007. 50(6):1148-1155. Available from: http://onlinelibrary.wiley.com/o/cochrane/clcentral/articles/414/CN00609414/frame.html

22. Rosenstock J, Rendell MS, Gross JL, Fleck PR, Wilson CA, Mekki Q. Alogliptin added to insulin therapy in patients with type 2 diabetes reduces $\mathrm{HbA1c}$ without causing weight gain or increased hypoglycaemia. Diabetes Obesity Metab. 2009;11(12):1145-1152. doi:10.1111/dom.2009.11.issue-12

23. Vilsbøll T, Rosenstock J, Yki-Järvinen H, et al. Efficacy and safety of sitagliptin when added to insulin therapy in patients with type 2 diabetes. Diabetes Obes Metab. 2010;12(2):167-177. Available from: http://online library.wiley.com/o/cochrane/clcentral/articles/573/CN-00769573/frame. html
24. Barnett AH, Charbonnel B, Donovan M, Fleming D, Chen R. Effect of saxagliptin as add-on therapy in patients with poorly controlled type 2 diabetes on insulin alone or insulin combined with metformin. Curr Med Res Opin. 2012. 28(4):513-523. Available from: http://onlinelibrary.wiley. com/o/cochrane/clcentral/articles/728/CN-00842728/frame.html

25. Hong ES, Khang AR, Yoon JW, et al. Comparison between sitagliptin as add-on therapy to insulin and insulin dose-increase therapy in uncontrolled Korean type 2 diabetes: CSI study. Diabetes Obes Metab. 2012;14 (9):795-802. Available from: http://onlinelibrary.wiley.com/o/cochrane/ clcentral/articles/500/CN-00842500/frame.html

26. Kadowaki T, Tajima N, Odawara M, et al. Efficacy and safety of sitagliptin add-on therapy in Japanese patients with type 2 diabetes on insulin monotherapy. Diabetol Int. 2013;4(3):160-172. doi:10.1007/ s13340-013-0109-z

27. Kothny W, Foley J, Kozlovski P, Shao Q, Gallwitz B, Lukashevich V. Improved glycaemic control with vildagliptin added to insulin, with or without metformin, in patients with type 2 diabetes mellitus. Diabetes Obesity Metab. 2013;15(3):252-257. doi:10.1111/dom.12020

28. Yki-Järvinen H, Rosenstock J, Durán-Garcia S, et al. Effects of adding linagliptin to basal insulin regimen for inadequately controlled type 2 diabetes: a $\geq 52$-week randomized, double-blind study. Diabetes Care. 2013;36(12):3875-3881. doi:10.2337/dc12-2718

29. Kaku K, Mori M, Kanoo T, Katou M, Seino Y. Efficacy and safety of alogliptin added to insulin in Japanese patients with type 2 diabetes: a randomized, double-blind, 12-week, placebo-controlled trial followed by an open-label, long-term extension phase. Expert Opin Pharmacother. 2014. 15(15):2121-2130. Available from: http://onlinelibrary.wiley.com/ o/cochrane/clcentral/articles/221/CN-01014221/frame.html

30. Hirose T, Suzuki M, Tsumiyama I. Efficacy and safety of vildagliptin as an add-on to insulin with or without metformin in japanese patients with type 2 diabetes mellitus: a 12-week, double-blind, randomized study. Diabetes Ther. 2015. 6(4):559-571. Available from: http://onlinelibrary.wiley.com/o/cochrane/clcentral/articles/ 281/CN-01125281/frame.html

31. Linjawi S, Sothiratnam R, Sari R, Andersen H, Hiort LC, Rao P. The study of once- and twice-daily biphasic insulin aspart 30 (BIAsp 30) with sitagliptin, and twice-daily BIAsp 30 without sitagliptin, in patients with type 2 diabetes uncontrolled on sitagliptin and metformin - The Sit2Mix trial. Prim Care Diabetes. 2015. 9(5):370-376. Available from: http://onlinelibrary.wiley.com/o/cochrane/clcentral/ articles/453/CN-01097453/frame.html

32. Mathieu C, Shankar RR, Lorber D, et al. A randomized clinical trial to evaluate the efficacy and safety of co-administration of sitagliptin with intensively titrated insulin glargine. Diabetes Ther. 2015;6 (2):127-142. Available from: http://onlinelibrary.wiley.com/o/ cochrane/clcentral/articles/457/CN-01083457/frame.html

33. Otsuka Y, Yamaguchi S, Furukawa A, Kosuda M, Nakazaki M, Ishihara H. Addition of sitagliptin or metformin to insulin monotherapy improves blood glucose control via different effects on insulin and glucagon secretion in hyperglycemic Japanese patients with type 2 diabetes. Endocr J. 2015. 62(2):133-143. Available from: http://online library.wiley.com/o/cochrane/clcentral/articles/243/CN-01052243/ frame.html

34. Sato S, Saisho Y, Kou K, et al. Efficacy and safety of sitagliptin added to insulin in Japanese patients with type 2 diabetes: the EDIT randomized trial. PLoS One. 2015;10(3):e0121988.

35. Takahashi H, Sakai K, Kawanishi K, et al. Efficacy of switching from premix analog insulin twice daily injection to insulin glargine once daily injection with sitagliptin. Diabetol Int. 2015;6(1):33-38. Available from: http://onlinelibrary.wiley.com/o/cochrane/clcentral/ articles/123/CN-01069123/frame.html

36. Mita T, Katakami N, Shiraiwa T, et al. Sitagliptin attenuates the progression of carotid intima-media thickening in insulin-treated patients with type 2 diabetes: the sitagliptin preventive study of intima-media thickness evaluation (SPIKE): a randomized controlled trial. Diabetes Care. 2016;39(3):455-464. doi:10.2337/dc15-2145 
37. Ning G, Wang WQ, Li L, et al. Vildagliptin as add-on therapy to insulin improves glycemic control without increasing risk of hypoglycemia in Asian, predominantly Chinese, patients with type 2 diabetes mellitus. $J$ Diabetes. 2016;8(3):345-353. doi:10.1111/1753-0407.12303

38. Cao Y, Gao F, Zhang Q, et al. Efficacy and safety of coadministration of sitagliptin with insulin glargine in type 2 diabetes. J Diabetes. 2017;9(5):502-509. doi:10.1111/1753-0407.12436

39. Kadowaki T, Kondo K, Sasaki N, et al. Efficacy and safety of teneligliptin add-on to insulin monotherapy in Japanese patients with type 2 diabetes mellitus: a 16-week, randomized, double-blind, placebo-controlled trial with an open-label period. Expert Opin Pharmacother. 2017;18 (13):1291-1300. doi:10.1080/14656566.2017.1359259

40. Kadowaki T, Muto S, Ouchi Y, Shimazaki R, Seino Y. Efficacy and safety of saxagliptin in combination with insulin in Japanese patients with type 2 diabetes mellitus: a 16-week double-blind randomized controlled trial with a 36-week open-label extension. Expert Opin Pharmacother. 2017;18 (18):1903-1919. doi:10.1080/14656566.2017.1379990

41. Kanazawa I, Tanaka KI, Notsu M, et al. Long-term efficacy and safety of vildagliptin add-on therapy in type 2 diabetes mellitus with insulin treatment. Diabetes Res Clin Pract. 2017;123:9-17. Available from: http:/onlinelibrary.wiley.com/o/cochrane/clcentral/ articles/601/CN-01301601/frame.html

42. Shankar RR, Bao YQ, Han P, et al. Sitagliptin added to stable insulin therapy with or without metformin in Chinese patients with type 2 diabetes. J Diabetes Investig. 2017;8(3):321-329. doi:10.1111/jdi.12585

43. Chen C, Yu Q, Zhang S, Yang P, Wang CY. Assessing the efficacy and safety of combined DPP-4 inhibitor and insulin treatment in patients with type 2 diabetes: a meta-analysis. Int J Clin Exp Pathol. 2015;8(11):14141-14150.

44. Kim YG, Min SH, Hahn S, Oh TJ, Park KS, Cho YM. Efficacy and safety of the addition of a dipeptidyl peptidase-4 inhibitor to insulin therapy in patients with type 2 diabetes: a systematic review and meta-analysis. Diabetes Res Clin Pract. 2016;116:86-95. doi:10.1016/j.diabres.2016.03.011
45. Moher D, Liberati A, Tetzlaff J, Altman DG. Preferred reporting items for systematic reviews and meta-analyses: the PRISMA statement. BMJ. 2009;339:b2535. doi:10.1136/bmj.b2651

46. Higgins J, Green S. Cochrane handbook for systematic reviews of interventions version 5.1.0. Cochrane Collab. 2011. Available from: www.cochranehandbook.org

47. Higgins JP, Thompson SG, Deeks JJ, Altman DG. Measuring inconsistency in meta-analyses. BMJ. 2003;327(7414):557-560. doi:10.1136/bmj.327.7414.557

48. Egger M, Davey Smith G, Schneider M, Minder C. Bias in metaanalysis detected by a simple, graphical test. BMJ. 1997;315 (7109):629-634. doi:10.1136/bmj.315.7109.629

49. Cho YM, Merchant CE, Kieffer TJ. Targeting the glucagon receptor family for diabetes and obesity therapy. Pharmacol Ther. 2012;135 (3):247-278. doi:10.1016/j.pharmthera.2012.05.009

50. Yu M, Shankar RR, Zhang R, et al. Efficacy and safety of sitagliptin added to treatment of patients with type 2 diabetes inadequately controlled with premixed insulin. Diabetes Obes Metab. 2019;21 (2):408-411. doi:10.1111/dom.13517

51. Min SH, Yoon JH, Hahn S, Cho YM. Comparison between SGLT2 inhibitors and DPP4 inhibitors added to insulin therapy in type 2 diabetes: a systematic review with indirect comparison meta-analysis. Diabetes Metab Res Rev. 2017;33(1):e2818. doi:10.1002/ dmrr.v33.1

52. Yang W, Cai X, Gao X, Chen Y, Chen L, Ji L. Addition of dipeptidyl peptidase-4 inhibitors to insulin treatment in type 2 diabetes patients: a meta-analysis. J Diabetes Investig. 2018;9(4):813-821. doi:10.1111/jdi.12764

\section{Publish your work in this journal}

Diabetes, Metabolic Syndrome and Obesity: Targets and Therapy is an international, peer-reviewed open-access journal committed to the rapid publication of the latest laboratory and clinical findings in the fields of diabetes, metabolic syndrome and obesity research. Original research, review, case reports, hypothesis formation, expert opinion and commentaries are all considered for publication. The manuscript management system is completely online and includes a very quick and fair peer-review system, which is all easy to use. Visit http://www.dovepress.com/testimonials.php to read real quotes from published authors. 